\title{
PROPERTIES OF MYELINATED FIBERS IN FROG SCIATIC NERVE AND IN SPINAL CORD AS EXAMINED WITH MICRO-ELECTRODES*
}

\author{
ICHIJI TASAKI \\ Central Institute for the Deaf St. Louis, Mo. U.S.A.
}

In 1951, during the period from February to May, Huxley and I made a series of observations, using glass micro-electrodes (Ling and Gerard, 1949; Nastuk and Hogkin, 1950), on the action potentials recorded from inside the frog motor nerve fiber. A large motor fiber was isolated from a sciatic-gastrocnemius preparation, and the operated region of the preparation, immersed in Ringer's fluid, was brought under a polarizing microscope with an objective lens of water-immersion type. A micro-electrode of below 0.5 micron in diameter, held by means of a micro-manipulator, was pushed into the nerve fiber, and the potential difference between the intra-cellular electrode and the outside fluid medium was recorded with a cathode-ray oscillograph used in conjunction with a DC amplifier. Action potentials were elicited from the fiber by induction shocks applied to the proximal nerve trunk. Muscular contractions were taken as an index of the propagation of impulses across the impaled region. The main features of the results obtained are as follows :

1. When the tip of the micro-electrode was just under the layer of myelin or when a dimpling of myelin into the axis-cylinder was seen at the tip of the micro-electrode, there was practically no measurable resting potential. But a propagated nerve impulse could very often evoke sizable monophasic action potentials (generally not exceeding $30 \mathrm{mV}$ in peak value). At this stage, propagation of impulses across the impaled region was not impaired.

2. When the tip of the micro-electrode was introduced deep inside the axis-cylinder, a resting potential of 10 to $40 \mathrm{mV}$ was observed. The action potentials observed at this stage were large at the beginning (20 to $40 \mathrm{mV}$ ), but soon a notch appeared at the peak of the action potential. As time elapsed, this notch became more pronounced, and generally within 2 to 5 minutes after introduction of the micro-electrode, a stage was reached at which the part of action potential following the notch suddenly disappeared. Propagation across the impaled region was blocked at this moment.

The first part of the present investigation is an extension of the abovestated experiments done in England. In order to correlate the action potentials observed with an internal electrode with the action currents recorded with the

Received for publication September 22, 1952.

* This work was carried out under Contract Nóonr-272 between the Office of Naval Research and Central Institute for the Deaf. 
partition method (Tasaki and Takeuchi, 1941; Huxley and Stämpfli, 1950), the longitudinal currents flowing through the axis-cylinder and the potentials of the axis-cylinder was recorded simultaneously at the same internode. The results obtained are somewhat different from those obtained by Woodbury (1952) who recorded full-sized resting and action potentials from undissected fibers in the nerve trunk.

Next, a method was developed by which a constant current for polarizing the nerve fiber could be sent into the same micro-electrode that was measuring the action potential. It was also found possible to excite single fibers by stimulating currents applied through an intra-cellular electrode.

Finally, using micro-electrodes, electrical responses were recorded from individual fibers in the dorsal column of the frog spinal cord. The general character of the action potentials observed was much the same as that of the action potentials recorded by K. Frank* (personal communication) from individual fibers in cat spinal cord by the same technique. By the method of polarization with an internal electrode and also by measuring the variation in threshold along a fiber in the most superficial layer of the dorsal funiculus, direct physiological evidence was obtained indicating that there are nodes of Ranvier in the central nervous system and they play the same role as in peripheral nerves.

\section{METHODS}

1. Micro-electrode. Since the myelinated nerve fibers of the frog are 2 to 12 microns in diameter, recording action potentials from inside these fibers is more difficult than recording from muscle fibers of 100 microns diameter (Nastuk and Hodgkin, 1950). In those large skeletal muscle fibers a considerable length of the micro-electrode near its tip can be introduced into the protoplasm. Hence, the capacitative flow of current through the extremely thin wall of the microelectrode is considered to play some role in improving the response of this high resistance electrode to rapidly changing voltages. With isolated single nerve fibers, however, it seemed highly probable that such leakage of current through the wall of the micro-electrode reduces the response of this electrode to high frequency alternating currents.

With a view to examining this point, the following simple observation was made (fig. 1). A micro-electrode, drawn from glass tubing about $1 \mathrm{~mm}$. in outside diameter and about $0.2 \mathrm{~mm}$. thick to a tip of about one fourth of a
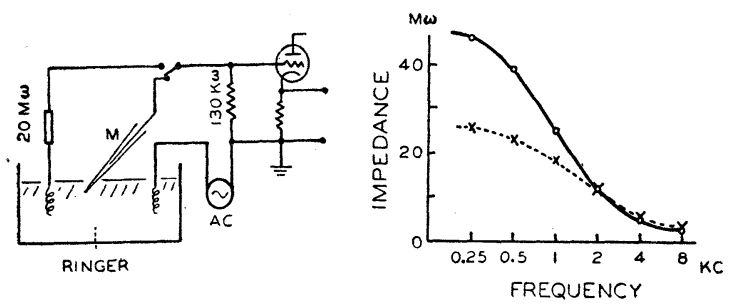

FIG. 1. Experimental arrangement used for measuring electric impedance of a micro-electrode (left), and two examples of the results of such measurements (right).

* National Institutes of Health, Bethesda, Md., U.S.A. 
micron and filled with a 3 molar potasium chloride solution, was held with a small clamp made of plastic and its tip was immersed in frog Ringer's solution. A piece of silver wire of 100 microns in diameter and $15 \mathrm{~cm}$. long was used for connecting the grid of the cathode-follower and the electrode. Another piece of silver wire was immersed in the fluid (in the Petri dish), and an AC voltage of about $150 \mathrm{mV}$ peak-to-peak was applied between the ground and the wire immersed in the dish. The impedance of the micro-electrode was determined by measuring the voltage across a low resistance $(130 \mathrm{kilohms})$ inserted in the circuit.

The diagram on the right-hand side of fig. 1 shows two examples of such measurements. The impedance at 250 cycles per second agreed fairly well with the $\mathrm{DC}$ resistance of the electrode. As the frequency went up, the impedance fell rapidly, and at about 5000 cycles per second it reached a value of $1 / 10$ to $1 / 20$ of the DC resistance. When a commercial resistor of 20 megohms replaced the micro-electrode, a fairly constant value was obtained up to this frequency. Therefore the measurements are reliable in this frequency range.

These measurements indicate that, in recording action potentials of a nerve fiber with a micro-electrode, leakage of current through the wall of the electrode can actually cause a considerable distortion in shape and a reduction in the absolute value ( $c f$. Woodbury, 1952). When the tip of a micro-electrode is immersed in ringer, a nearly stationary state is reached in the electrode very soon, the $\mathrm{KCl}$ solution near the tip of the electrode being diluted by Ringer. The high DC resistance of such a micro-electrode is due mainly to the low electrolyte concentration near the tip. (Note that $\mathrm{NaCl}$ in frog ringer is approximately 0.12 molar.) Since the impedance of the electrode does not increase appreciably during the course of measurement (5 to 100 minutes), it is clear that the region of high specific resistance (namely low electrolyte content) is localized in the region near the very tip of the micro-electrode where the glass wall is extremely thin. Capacitative flow of current through this part of the electrode, which undoubtedly accounts for the curves in fig. 1, right, gives us a considerable difficulty in determining the absolute value and the exact timecourse of the action potentials recorded with this type of internal electrode.

2. Cathode-follower, amplifier and cathode-ray oscillograph. In early experiments electric responses of a single nerve fiber were recorded simultaneously with three independent channels of amplifier and cathode-ray oscillograph. The first channel was used for recording action potentials from inside the fiber with a micro-electrode, the second channel for photographing action currents flowing through the axis-cylinder, and the third channel for recording DC potentials from inside the fiber. The difference between the first and third channels is simply that the third channel has a lower voltage sensitivity but a DC amplifier so that it records resting potentials as well as action potentials. Each of these amplifiers was preceded by a cathode-follower stage, the vacuum tube used being RCA 1620 in triode connection (approximately $4 \times 10^{-11}$ ampere grid current). In many of the later experiments, only one or two of these three channels were used for recording responses. In all the records presented 
in this paper, movement of the electron beam was upward for a positive shift in potential at the input of the cathode-follower.

In recording electrical responses from single nerve fibers, the distinction should be made between a "current recording" and a "potential recording." We speak of a "current recording," when the two lead-off electrodes are shunted with a low resistor, far lower than that of the material to be examined, and consequently the recorded potential difference across this resistor is simply proportional to its resistance. Since the resistance of the fiber mounted across a bridge-insulator is of the order of 30 megohms, the grid resistance of 130 kilohms used in the present experiments is actually far lower than that of the material. In such cases the absolute value of the current is obtained by dividing the observed potential difference by the grid resistance. A low grid resistance raises the noiseto-signal ratio, but it serves to reduce the effect of capacity of the lead wire, which otherwise tends to make the frequency characteristic of the system poor on the high frequency side.

In "potential recording," the input impedance of the recording system should be far greater than that of the material to be examined. Actually, in recording action potentials of a nerve fiber with a micro-electrode, it is practically impossible to have this condition satisfied, because the effective input impedance is always lowered by the capacity between the lead wire and the ground and also by the leakage of current through the wall of the micro-electrode. The absolute values of action potentials obtained by this method, therefore, have very little physiological meaning.

3. Nerve fibers and bridge-insulator. Single fibers were obtained from sciatic-gastrocnemius preparations of the frog (rana esculenta) by the routine method (Tasaki, 1939). A bridge-insulator was made from two pieces of glass, each approximately $4 \times 7 \mathrm{~cm}$. wide and held with plastic plates at a distance of approximately $0.1 \mathrm{~mm}$. (Diagrams $A, B$ and $C$ in fig. 2). The fiber was laid across this 100-micron air-gap with the precautions described previously (p. 369, bottom, in Tasaki, 1939). Then the preparation was brought under a microscope ( 200 to $650 \times$ magnification) and the first node of Ranvier on the proximal side of the air gap $\left(N_{1}\right)$ was brought in the middle of the visual field. As the distance from the objective lens of the microscope to the fiber was $1.5 \mathrm{~mm}$., there was an ample space for handling the micro-electrode under the lens. The micro-electrode was pushed into the fiber in the region within \pm 200 microns from the proximal node. All the experiments were done at room temperature, $25^{\circ}$ to $26^{\circ} \mathrm{C}$.

4. Spinal cord. Spinal cord preparations were obtained by decapitating frogs and opening the vertebral column from behind. This was done after all the organs in the chest and abdomen and all the muscles in the limbs and the dorsum had been removed. The nerve fibers in the dorsal funiculus continued to carry impulses for more than two hours without blood supply (at $25^{\circ} \mathrm{C}$.). The exposed spinal cord was kept in a bath of Ringer's fluid.

For recording action potentials from fibers in the dorsal funiculus, a microelectrode was pushed into the cord diagonally in the fluid medium (fig. $2, D$ ). 


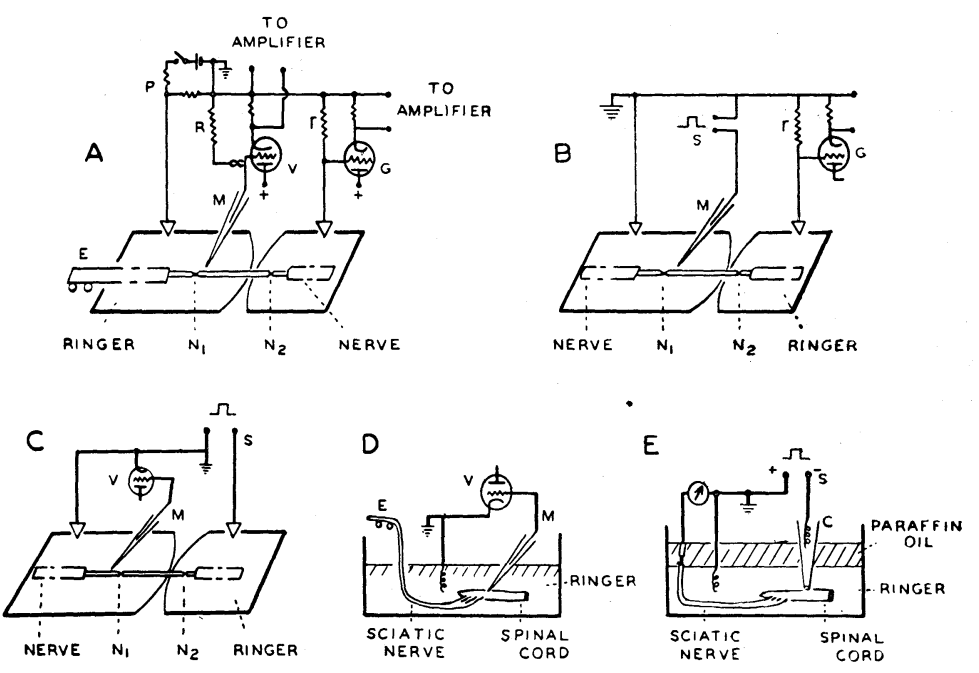

FIG. 2. Experimental arrangements used for simultaneous recording of longitudinal action currents and action potentials from inside a nerve fiber $(A)$, for stimulating a nerve fiber with an internal electrode $(B)$, for recording subthreshold responses of a node $(C)$, for recording action potentials from inside individual fibers in the spinal cord $(D)$ and for measuring thresholds of a fiber in the superficial layer of the spinal cord $(E)$. $P$ stands for a polarizing circuit, $R$ for a high resistance (120 megohms), $r$ for a low resistance $(0.13$ megohm), $V$ for the first stage of a potential recording device, $G$ for the first stage of a current recording system, $M$ for a micro-electrode, $E$ for a pair of stimulating electrodes, $S$ for the output of a square wave generator, $C$ for a relatively large glass capillary and $N$ 's for nodes of Ranvier of the single nerve fiber. All the diagrams are drawn out of proportion.

This was done under a binocular microscope $(20 \times)$. The fluid in the dish was grounded, and the sciatic nerve was stimulated with induction shocks from a transformer with an ungrounded secondary coil.

For threshold measurements on the fibers in the dorsal funiculus, a glass capillary of approximately 25 microns inside diameter was used to apply rectangular current pulses to the surface of the spinal cord. The capillary was filled with frog ringer and a fine silver wire, wound in a spiral at the end, conveyed the stimulating pulses from the output of a multi-vibrator to the capillary. The resistance of such a stimulating electrode was approximately 500 kilohms. The index of nerve excitation was single fiber responses led off from a group of approximately ten fibers entering the gastrocnemius muscle. Since only those fibers situated in the most superficial layer of the dorsal column gave responses on stimulation, many of those ten fibers did not give any response. To avoid possible contamination by reflex responses in the efferent nerve fibers, the 7 th, 8 th and 9 th ventral roots were cut. The operated region of the muscle nerve was suspended in the layer of paraffin oil floating above the Ringer's fluid (fig. 2,E). The distal portion of the nerve was led to an amplifier with an input resistance of approximately 1 megohm. 
RESULTS

\section{Part I. Fibers in the Peripheral Nerve}

1. Action potentials recorded from inside the myelin sheath. In this series of experiments, the experimental set-up shown in fig. $2, A$, was used. The fiber was so mounted on the bridge-insulator that the proximal two-thirds of the internode to be examined was immersed in the Ringer's fluid on the proximal side of the air-gap. While attempts were being made to push a micro-electrode into the layer of myelin, induction shocks were applied to the proximal nerve trunk at a rate of 3 to 4 per sec. By means of a loudspeaker operated by the output of the first (voltage) channel, sudden changes in potential of the micro-electrode were made audible. A micro-electrode with a DC resistance of 20 to 40 megohms was introduced into the fiber at a point 20 to 200 microns away from the first node on the proximal side of the bridge-insulator. The electrode was held horizontal and was brought to the fiber from the side. The tip of the electrode was pushed against the surface of the fiber, not perpendicularly, but at a relatively sharp angle (from 20 to 40 degrees between the axis of the fiber and the electrode). This generally resulted in a dimpling of the myelin layer into the axis-cylinder.

Generally when the dimpling of the myelin layer into the axis-cylinder was slight, neither a resting potential nor an action potential was observed on the oscillograph. But sometimes it happened that a small action potential, below $5 \mathrm{mV}$ peak value, recurred at the frequency of stimulation. When this small response appeared there was practically no observable change in the time-course of the action current recorded from the same internode (see fig. 3 , record $A$ ).

When the micro-electrode was pushed still further into the axis-cylinder, relatively large action potentials, generally 5 to $20 \mathrm{mV}$ peak value, appeared without any noticeable change in the action current of the same internode $(G)$ or in the DC potential of the micro-electrode (fig. $3, B$ ).

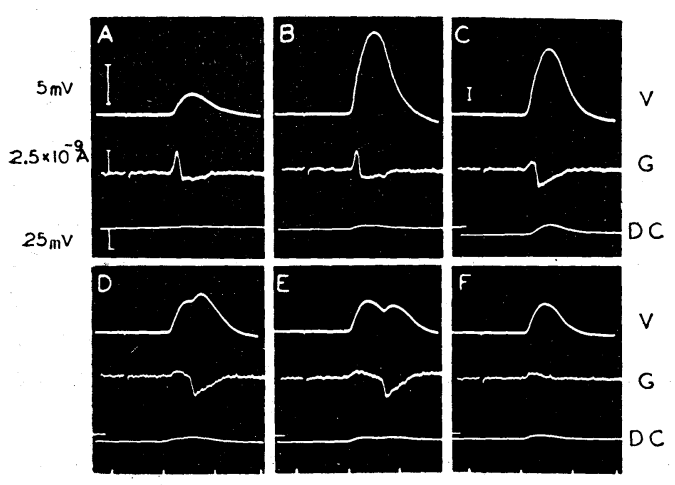

FIG. 3. Simultaneous records of action potentials $(V)$ with an internal electrode, longitudinal action currents. $(G)$ and resting potentials $(D C)$ of a single nerve fiber. Record $A$ was taken when the micro-electrodes, pushed into the mylin sheath at a point about 150 microns distal to the node, began to pick up action potentials. $B$ was taken after the electrode had been pushed a little deeper into the axis-cylinder. In $C$, the micro-electrode went still deeper and a resting potential appears in this record. The amplification for the uppermost channel is reduced by $10 \mathrm{db}$. $D$ to $F$ show progressive changes in the records during the period of about two minutes. Time markers, $1 \mathrm{msec}$. 
Then a further push or a slight jiggling of the micro-electrode caused sometimes a sudden shift in the base-line of the DC channel (fig. 3, C). At the moment when this sudden shift took place the observed action potential was largest ( 20 to $40 \mathrm{mV}$ peak value). This stage was immediately followed by a progressive deterioration both in the action potential and in the action current.

As the action potential recorded from inside the fiber became progressively smaller, a notch appeared near the peak of the response. As time elapsed this notch became clearer and clearer (fig. $3, D$ and $E$ ). The action current records taken simultaneously from the same internode (fig. 2, channel $G$ ) indicated very clearly that this appearance of a notch was due to delay in conduction across the internode under investigation. At the moment when a clear notch appeared on the potential $(V)$ channel, a reversal in the direction of the longitudinal current was always observed. Since this reversal of the longitudinal current is a sign of the spread of the physiologically active region to the distal side of the bridge-insulator (Tasaki and Takeuchi, 1941), there is no doubt that the start of the second hump following the notch is due to the activity of the node $\left(N_{2}\right.$ in fig. 2) on the distal side of the bridge-insulator.

At variable time ( 1 to 10 minutes) after the appearance of the resting potential in the third DC channel, the second hump in the action potential record dropped out completely. The simultaneous record of action currents showed that nervous conduction was blocked at this moment.

The conduction block was always preceded by a visible change in the shape of the nearest node of Ranvier $\left(N_{1}\right.$ in fig. 2). It was a surprise to see that a nerve impulse could travel across a node at which the gap in the myelin sheath had become many times wider than normal.

There were also many cases in which a resting potential failed to appear at all. This apparently shows that a thin layer of myelin over the tip of the micro-electrode is capable of maintaining a large DC potential (due to its high DC resistance). The tip of the electrode covered with a thin film of myelin can pass completely through axis-cylinder and reach the myelin sheath again before the film is perforated. It was my impression that in the present series of experiments the resting potential failed to appear more frequently than in the previous experiments done with Huxley in a cold British winter. It may be that the difference in room temperature caused this difference by changing the viscosity or the stiffness of the myelin.

2. Recording action potentials from inside a node of Ranvier. Although the gap between the myelin sheath at the node is generally a little less than 1 micron in width, it is possible to introduce a micro-electrode right into this naked portion of the axis-cylinder. It is, however, difficult to obtain large action potentials from this part of the fiber. The injury current arising from perforation of the nodal membrane seems to depress the activity of the fiber very rapidly.

Figure 4 gives an example of such observations. Record $A$ in this figure shows the time-course of the action current taken across the bridge-insulator. This typical binodal configuration has been described previously (Tasaki and Take- 


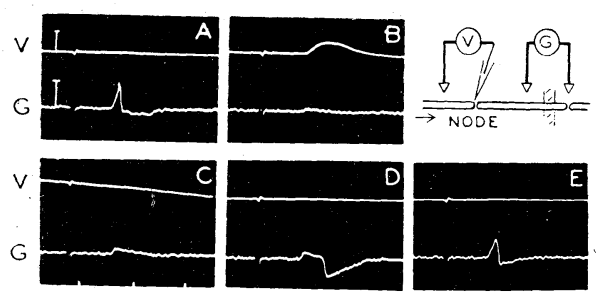

FIG. 4. Effect of a micro-electrode pushed into a node of Ranvier. $A$ : electrode still in Ringer around the fiber. $B$ : electrode in the node. $C$ : record taken while the electrode was being withdrawn; the disturbance in the upper record $(V)$ is due to movement of the electrode. $D:$ immediately after withdrawal of the electrode. $E$ : three minutes after withdrawal. Note the quick recovery in the longitudinal action current $(G)$. The bar at the top subtends $2.5 \mathrm{mV}$; the next bar for the action current records, $2.5 \times 10^{-9} \mathrm{~A}$. Time markers, 1 msec.

uchi, 1941). When a micro-electrode was pushed into the node, an action potential associated with a very slight resting potential (about $9 \mathrm{mV}$ in this case) was observed (Record $B$ ). At this moment the longitudinal action current was already very small, indicating that the node into which the electrode had just been inserted was practically inexcitable. Then the micro-electrode was pulled out (record $C$ ). This almost immediately (within $0.3 \mathrm{sec}$. in this case) restored nervous conduction across this node (record $D$ ). About three minutes later, the response of the injured node recovered almost to normal.

The fact that a micro-electrode inserted into a node of Ranvier caused a quick deterioration of the resting and the action potentials may reasonably be attributed to lack of the sealing effect of myelin. Where myelin is present it apparently reduces the leakage of current through the hole made by the puncture. The rapid recovery in the function of the node after removal of the electrode indicates that the structure of the nodal membrane is not rigid.

3. Polarization of a nerve fiber with an internal electrode. To send a constant current into a nerve fiber through a micro-electrode, a simple device illustrated in fig. 2, $A$, was used. The resistance, $R$, connected between the grid of the cathode-follower and the ground (120 megohms in most cases) reduced the observed size of the action potential slightly. But the absolute value of the observed potential had only little physiological meaning, and, since the noise level of our action potential record was still low enough, this reduction in the spike height did not cause any serious trouble in our recording. Now, closure of the polarizing circuit, $P$ in the figure, caused a current which flowed through the micro-electrode and traversed the surface membrane of the nerve fiber.

Attention should be called in this procedure to the fact that there is a potential difference between the silver wire in the micro-electrode (with 3 molar $\mathrm{KCl}$ in it) and the ground electrode (a silver wire in Ringer-agar gel). The micro-electrode was generally 60 to $80 \mathrm{mV}$ negative relative to the ground electrode. The resting potential of the nerve fiber also caused a constant current through the grid resistance, $R$, when the micro-elctrode was inserted into the fiber. 
The experimental procedure of this series of experiments was as follows: First, a single nerve fiber was mounted on a bridge-insulator and, with the tip of the micro-electrode kept in Ringer's fluid, a record of action current was taken (fig. 5, top left). At this time, the resistance ( $R$ in the figure) was left disconnected and the polarizing voltage $(P)$ was adjusted to cancel the electrode potential. Then the micro-electrode was pushed gradually into the myelin-covered portion of the fiber until a resting potential appeared on the DC channel. The responses of the fiber to induction shocks applied to the proximal nerve trunk deteriorated gradually, as was described before. Next the resistance $(R)$ was connected to the grid of the cathode-follower, and, by changing the polarizing voltage $(P)$, direct currents of varying intensities were sent into the micro-electrode (the lower column of fig. 5).

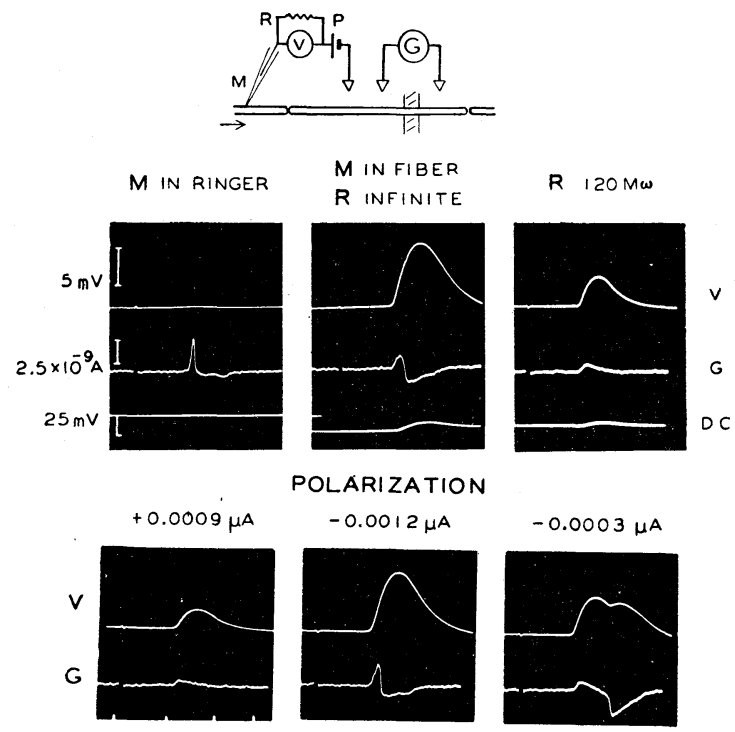

FIG. 5. Effect of constant currents sent into a nerve fiber through a micro-electrode. A positive polarizing current is a current that flows outwards through the tip of the microelectrode. Time markers, 1 msec.

When a current was made to flow outward through the micro-electrode inserted in the axis-cylinder, namely when the tip of the electrode behaved as a source of current, responses of the fiber, which were already small due to the progressive deterioration, became still smaller. With currents flowing the opposite direction, namely with the micro-electrode behaving as a sink of current, recovery in the size of response was always observed. The intensity of current given in the figure was calculated simply by dividing the shift in the polarizing voltage $(P)$ by the total resistance in the circuit (the sum of $R$ and the DC resistance of the micro-electrode). At a certain adequate intensity, just critical conduction of impulses across this impaled region was always observed.

It was mentioned in the early part of this paper that, when the tip of the micro-electrode had just penetrated the myelin sheath, action potentials without any associated resting potential could be observed. Passage of a constant current through such an electrode covered with a thin film of myelin did not bring 
about any appreciable change in the size of responses. An increase in the polarizing voltage merely caused an irregular noise in the output.

4. Excitation of a nerve fiber with a micro-electrode. In stimulating a single nerve fiber by a current pulse applied through an internal electrode, leakage of current through the wall of the electrode, mentioned under "Method," again complicates to the time course of the applied current pulse. When the tip of the electrode is inserted deep in the nerve fiber, a rectangular voltage pulse applied to the electrode tends to send into the fiber a current pulse with a sharp initial peak. When, on the contrary, just the tip of the electrode is introduced in to the axis-cylinder, the leakage through the wall of the electrode tends to round off the initial phase of the current. The qualitative aspects of the experimental results obtained by this method, however, are still of some interest.

When a micro-electrode was pushed into the myelin-covered portion of a nerve fiber and action potentials without any associated resting potential were being observed through the electrode, the condition of the fiber was found to be stable enough to obtain a steady threshold value for a period of five to ten minutes. On such occasions, current pulses which were applied to the fiber with the micro-electrode connected to the anode of the stimulator (the cathode being connected to the surrounding fluid medium) gave clear responses in the current-recording channel $(G)$ connected to the same or to the adjacent internode. The threshold voltage measured by this method varied considerably from experiment to experiment, but with micro-electrodes of 20 to 40 megohm DC resistance, it was generally between 100 and $500 \mathrm{mV}$. As in the case of excitation with extra-cellular electrodes, the latency became shorter with increasing stimulus strength.

When the micro-electrode inserted in the myelin layer was connected to the cathode of the stimulating circuit, responses appeared only after the end of the applied voltage pulse (see the last record in fig. 6). The threshold for such a break excitation was much higher than that for a make excitation, and repeated excitation by such strong break stimuli caused gradual deteriora-

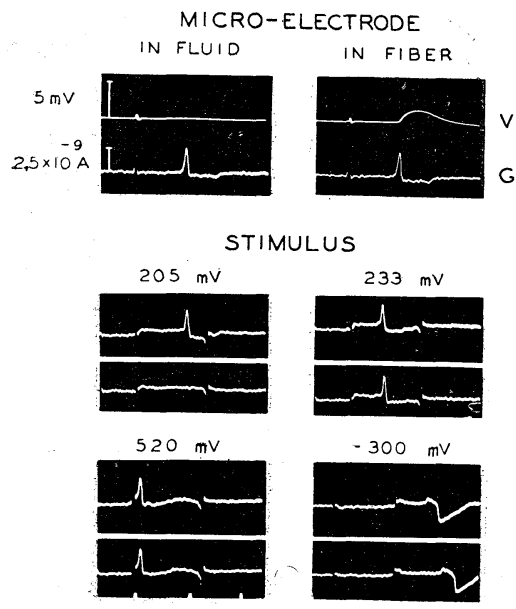

FIG. 6. Electric stimulation of a nerve fiber with an internal electrode. The micro-electrode was pushed into the myelin sheath at a point about 10 microns away from a node (proximal to $N_{1}$ in fig. 2). No resting potential was observed. Rectangular voltage pulses were applied between the micro-electrode and the fluid medium. The threshold stimulus strength was $205 \mathrm{mV}$; the two records in this column were obtained at interval of $0.3 \mathrm{sec}$. with the same stimulus intensity. The negative voltage in the last record indicates that the micro-electrode was now connected to the stimulating cathode. Time markers, $1 \mathrm{msec}$. apart. 
tion of the nerve responses.

The experiments in which the micro-electrode for stimulation was pushed into one of the nodes of Ranvier of the nerve fiber gave results quite different from what has been mentioned above. Since an electrode inserted directly into the node is causes rapid deterioration of the activity of the node, experiments were slightly difficult to perform and the results seemed confusing in the early stages of the experiments. But, when the following procedure was adopted, consistent results were obtained.

First, with the tip of the micro-electrode kept in Ringer's fluid, relatively strong voltage pulses ( 0.5 volt in most cases) were applied between the ground and the micro-electrode synchronously with the sweep of the oscillograph. Then the electrode was gradually brought close to the surface of the node, and photographic records were taken of the figures on the screen of the oscillograph while the electrode was being pushed into the node. The electrode was always withdrawn within one or two seconds after the start of responses on the oscillograph.

The results obtained by this method are as follows: Penetration of the tip of the micro-electrode through the nodal membrane is characterized by a sudden shift in the oscillograph beam during the period of current flow (see the three records on the lower left-hand corner of fig. 7). (This statement is justified by

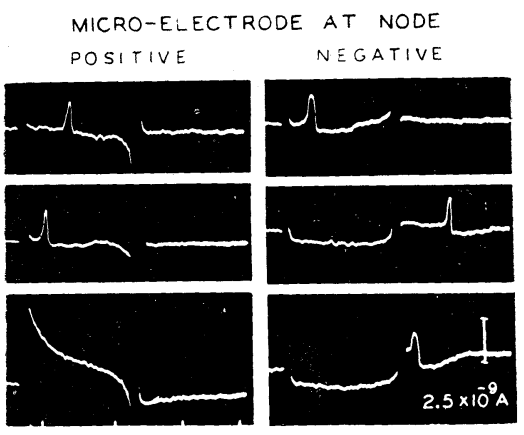

FIG. 7. Excitation of a nerve fiber with a micro-electrode pushed against or into the nodal membrane. The stimulus strength was $500 \mathrm{mV}$ throughout. Each one of these six records was taken in an independent attempt to penetrate the node with the stimulating electrode. For details, see the text. Time markers, 1 msec. apart.

the fact that the physiologically important nodal membrane has a high electric resistance. In these experiments resting potentials could not be measured, however, because the micro-electrode was connected to the stimulating circuit.) The nerve fiber begins to respond to the stimuli before the tip of the micro-electrode penetrates the nodal membrane. A node of Ranvier responds both to positive and to negative current pulses applied on its outer surface (see the two upper records in fig. 7). After the tip of the micro-electrode has pierced the nodal membrane, a current flowing outwards through the electrode elicits a response only during the period of current flow, and a pulse of current flowing in the opposite direction only after the end of the pulse.

In figs. 7 and 8 several examples of records obtained by the procedure described above are presented. The three upper records in fig. 7 and records $A$ and $B$ in fig. 8 show the responses obtained without any appreciable shift in 
the base-line during the period of current flow. The first upward movement of the oscillograph line in these responses indicates that the stimulating current is actually exciting primarily the node $\left(N_{1}\right)$ to which the currents are applied.

When the tip of the micro-electrode was inside the nodal membrane, a current pulse flowing inwards through the electrode caused a downward deflection, as shown by the two lower records in the right-hand column of fig. 7 . During the pulse there was no sign of activity, but, after the end of the pulse a response appeared at this node. With pulses of current flowing outward through the tip of the electrode inserted into the axis-cylinder through the nodal membrane, it was slightly difficult to demonstrate that there was a response during the period of current flow. The superimposed record $C$ in fig. 8 shows

MICRO-ELECTRODE AT NODE

POSITIVE

NEGATIVE
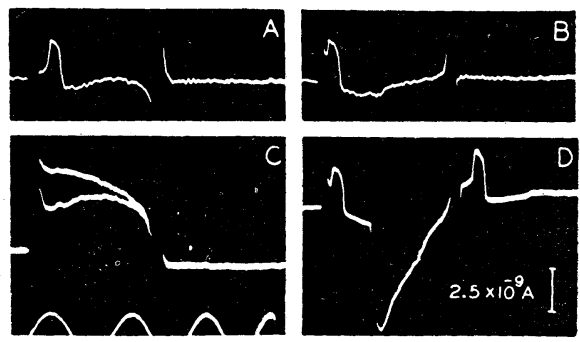

FIG. 8. The same as fig. 7 , but taken from a different preparation. Time marker, msec.

such a response. The lower curve in this record was taken within half a second after penetration of the electrode through the nodal membrane, and the upper curve approximately 2.5 seconds after the first recording. The difference between these two curves is considered to be due to a response which deteriorated very rapidly. Judging from the direction of the current, this difference is due mainly to the response of the node $N_{2}$ (fig. $2, B$ ) on the distal side of the bridgeinsulator. Apparently this distal node was excited by the action current from the node $N_{1}$ (which is completely masked by the shock artefact) and by direct spread of the stimulating current to $N_{2}$. The record at the left bottom of fig. 7 was taken apparently after deterioration of those responses.

Record $D$ in fig. 8 is one of the two rare records showing penetration of the electrode during the period of current flow. The first response in this photograph is the one induced while the tip was still outside the nodal membrane; it is a make-response to a cathodal (micro-electrode negative) stimulus. The sharp downward deflection which appears about half a millisecond after the start of the current pulse indicates that the penetration occurred at this moment. As the micro-electrode was negative in this case, another (break) response was initiated by the termination of the current pulse.

The above-mentioned finding that a node of Ranvier can be excited both by anodal and cathodal current pulses applied through a micro-electrode placed directly on its outer surface can reasonably be interpreted as follows: In this type of experiment, the short cylindrical surface of the axis-cylinder does not behave as a uniform functional unit. One part of the membrane is traversed 
by an outward-directed current, while the remaining part is subjected to a current flowing in the opposite direction. There is therefore very little net current flow spreading along the axis-cylinder toward the adjacent node. The part of the nodal membrane subjected to an outward-directed current can initiate a response which, by virtue of the process of restimulation by local circuits, eventually brings the whole nodal membrane into the active state.

The break excitation observed with the micro-electrode inserted in the myelin sheath or in the axis-cylinder should naturally be attributed primarily to the start of a transient current in the opposite direction on withdrawal of the imposed current, due to the capacities of the myelin sheath and of the nodal membrane.

5. Subthreshold responses. It has been shown by Takeuchi and myself (1942) that, in stimulation of a single nerve fiber, small electrical responses of variable sizes appear at the site of stimulation. These responses have a relatively small restimulating effect upon the adjacent node of Ranvier and they are not followed by any detectable refractoriness. According to our interpretation of the concept of "local response" first introduced by Rushton (1937), we examined whether these small responses could actually be a complete depolarization of a small area within one node of Ranvier. As the test we made gave negative results, we concluded that they could not be "local" responses.

According to Hodgkin, Huxley and Katz (1949), however, a "subthreshold" response in invertebrate nerve fibers (Hodgkin, 1937) is not an all-or-none response in a small area. It is followed by a period of supernormal excitability instead of a subnormal, refractory period. Since such a period of supernormal excitability exists also in the myelinated fiber, our previous explanation of the small subthreshold responses is, as has been pointed out by several British physiologists, certainly wrong. Later on, Tasaki and Mizuguchi (1948), Castillo and Stark (1951), Huxley and Stämpfli (1951), Schoeffli and Erlanger (1951), Woodbury (1952) and others demonstrated subthreshold responses under various experimental conditions.

Using the experimental set-up of fig. 2. $C$, an attempt was made to record subthreshold responses of a node simultaneously with current- and potentialrecording devices. A micro-electrode was introduced into the myelin-covered portion of the fiber, generally at a point 20 to 40 microns proximal to the node $N_{1}$ in fig. 2. Short rectangular voltage pulses (approximately $0.1 \mathrm{msec}$. duration) were applied at a frequency of 3 to 4 per second. The intensity of the shock was so adjusted that full-sized responses were obtained in 30 to 50 per cent of the trials. When the micro-electrode was pushed deep into the axiscylinder, namely, when the node was slightly depolarized by the puncture with the electrode but still capable of developing large action potentials, photographic records as shown in fig. 9 were often obtained. In this record, the full-sized action potential appeared simultaneously with the full-sized action current (which caused a downward deflection in the lower record), and the small, subthreshold action potential was associated with a small, subthreshold action current. 


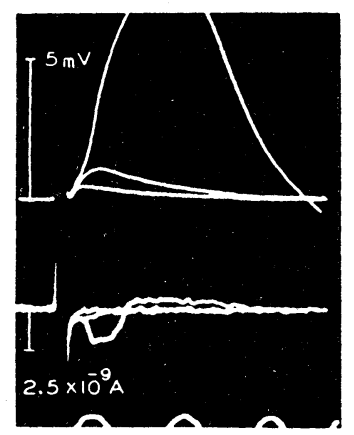

FIG. 9. Superimposed records show a subthreshold response of a node of Ranvier. The experimental set-up of figure 2, C, was used. The micro-electrode was pushed into the fiber at a point approximately 35 microns proximal to the node $N_{1}$. Time marker, 0.5 msec.

It should be mentioned in this connection that, in a fresh non-depolarized node, variable subthreshold action potentials were so small that they were almost undetectable by this very sensitive method. It is likely that in our previous experiments (1942) the node under investigation was in a slightly depolarized state which could give relatively large subthreshold responses.

6. Recording action potentials from small myelinated fibers. All the experiments stated up to this time were done on single large motor nerve fibers which were 9 to 12 microns in diameter. Since all these experiments showed that a micro-electrode was a powerful tool for recording action potentials from large nerve fibers, it seemed desirable to know whether or not this tool was applicable to smaller myelinated fibers. First, attempts were made to record action potentials from inside an isolated small motor nerve fiber (Tasaki and Kano, 1942) which is known to cause a slow muscular contraction and small monophasic muscle action potentials on stimulation. It was found easy to obtain a response of approximately $10 \mathrm{mV}$ from those fibers of 4 to 5 microns in diameter. Just as in large motor fibers, action potentials deteriorated when a resting potential appeared on the DC channel. When action potentials without any associated resting potential were observed, the fiber continued to conduct impulses for more than 30 minutes.

In fig. 10 is given an example of responses recorded from a small fiber. Three fibers entering the gastrocnemius muscle were isolated; their diameters were 11,4 and 3 microns respectively (measured under a low-power microscope). Action current records taken across a bridge-insulator (the lower records in the figure) show how the intensity of action current and the rate of conduction

FIG. 10. Simultaneous records of action potentials (taken with a micro-electrode) and action currents (led off by a bridge-insulator) from a three fiber preparation. Conduction distance, approximately $35 \mathrm{~mm}$. Time marker, msec.

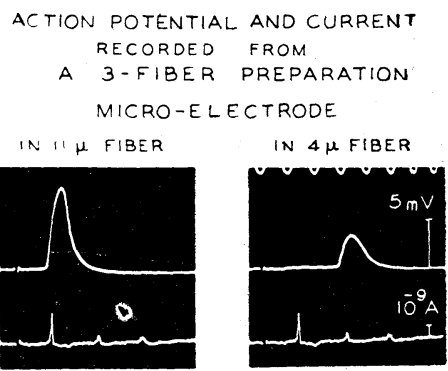


differed among these three fibers. A micro-electrode was first inserted into the largest fiber (left column in the figure). The action potential started almost simultaneously with the largest action current. Then the electrode was withdrawn and pushed into the smaller fiber. The action potential now started simultaneously with the second peak in the action current record (right column). All these observations showed very clearly that recording action potentials from 3 to 4 micron fibers with micro-electrodes was actually possible and practical.

Next, attempts were made to record single flber responses from an intact nerve trunk with a micro-electrode, just as in Woodbury's experiments. A nerve trunk was immersed in a bath of Ringer's fluid and a micro-electrode was pushed into the nerve through a small hole made in the epineurium. Unmistakable single-fiber responses were easily obtained on stiumlation of the nerve at one end. The responses from the surrounding fibers were not disturbing, because the fluid medium in the dish reduced the undesired potentials and the sensitivity of the amplifier could be kept low enough to avoid recording them. The properties of the nerve fibers examined by this method were not in any respect different from what has been seen in isolated single fibers. Working on the most proximal part of the sciatic nerve (plexus ischiococcygeus), it was possible to obtain single-fiber responses with a micro-electrode pushed through an intact epineurium.

Figure 11 shows an example of an unpleasant complication which was encountered in applying the micro-electrode technique to the whole nerve trunk. Responses from two different fibers were recorded with a single micro-electrode. Five records in this figure were taken in the order of sequence presented in the figure at varying stimulus strengths which can be seen from the different sizes of the shock artefacts. These were undoubtedly the responses of two different fibers, large and small, with the rates of conduction in the ratio of approximately 3.5 to 1 . Apparently the micro-electrode had penetrated entirely through the larger fiber and the tip had reached the neighboring smaller fiber. It was mentioned in the Method section of this paper that a potential change could be recorded through the thin wall of a micro-electrode.

The experimental data presented in this section indicate that a glass microelectrode is a powerful tool for recording single fiber responses from an undissected nerve trunk. But for a quantitative study on the properties of the myelinated fiber this method involves a number of difficulties which are hard to overcome. Woodbury (1952) states that he obtained resting potentials of more than 50 $\mathrm{mV}$ and action potentials of above $100 \mathrm{mV}$. He used an electronic device to improve the high-frequency characteristic of his recording system; but it is difficult to believe that his method of adding some potential to the

FIG. 11. Responses of two different nerve fibers in an intact sciatic nerve recorded with one micro-electrode.

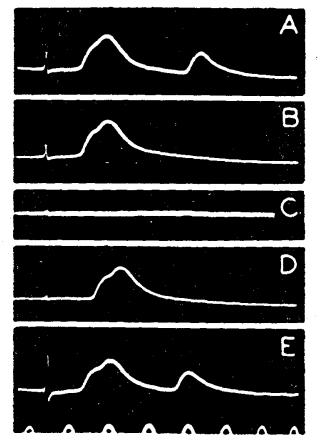


output of his amplifier is actually adequate for improving the characteristic of this complicated recording system. In our own experiments mentioned above (done on 33 isolated large motor fibers and a number of nerve trunks), no resting potential above $40 \mathrm{mV}$ was encountered.

\section{Part II. Fibers in the Dorsal Column of the Spinal Cord}

1. Recording action potentials from fibers in the dorsal funiculus. Action potentials from fibers in the dorsal funiculus were recorded with a micro-electrode pushed into the spinal cord through a hole made in the pia or sometimes with an electrode pushed through the pia. Stimuli (induction shocks) were applied to the proximal part of the sciatic nerve at a point approximately $30 \mathrm{~mm}$. away from the entry of the 7th spinal root. The whole spinal cord was immersed in Ringer's fluid in a Petri dish.

Five examples of the records obtained by this method are presented in fig. 12. In taking these records, the stimulus strength was adjusted to the threshold for the fiber from which the action potentials were recorded. As in the records taken from peripheral nerve fibers, spontaneous variations in the latency and occasional failure of response are seen in these records.
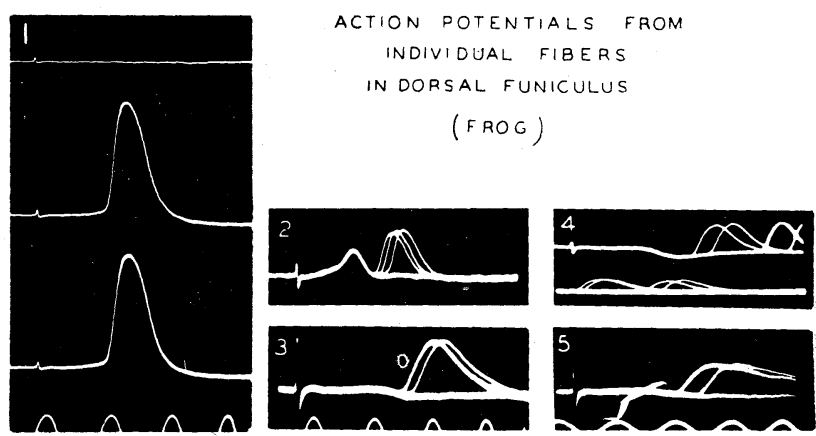

FIG. 12. Five examples of single-fiber responses obtained from the dorsal funiculus of the frog. Experimental set-up of fig. 2, D, was used. The spike heights of these responses are $6 \mathrm{mV}, 3.5 \mathrm{mV}, 3 \mathrm{mV}, 7 \mathrm{mV}$ and $1.5 \mathrm{mV}$ respectively (from preparation 1 to 5 ). Record number 4 shows an injury discharge arising at the region of the micro-electrode. Several sweeps are superimposed. Time markers, msec.

The peak value of the action potential obtained by this method ranged from 1.5 to $8 \mathrm{mV}$ (sampled from 21 fibers). This value seems slightly smaller than the corresponding value for the large motor nerve fiber. This would not be surprising, however, since the fibers in the dorsal funiculus are smaller than the motor fibers and micro-electrodes were introduced into those fibers without any visual control. The resting potential ranged from zero to $20 \mathrm{mV}$ when these action potentials were observed. Whenever there was a distinct resting potential, the action potential underwent a progressive deterioration. This deterioration of the action potential proceeded on some occasions fairly rapidly and on other occasions rather slowly (in 10 to 15 minutes). Action potentials without associated resting potential generally showed no progressive change within 10 to 15 minutes. 
The average rate of conduction along these fibers ranged from 15 to 25 $\mathrm{m} / \mathrm{sec}$. Since the major portion of the fiber is outside the spinal cord, the rate just mentioned indicates that we are here dealing with large and medium-sized (afferent) fibers in the sciatic nerve. The duration of the spike was in most cases something like one msec.; but in one occasion (record 5 in fig. 12) a much longer spike duration was observed.

2. Polarization of a single fiber in the dorsal funiculus. It was mentioned in the preceding section that when a resting potential was recorded together with the action potential, with a micro-electrode inserted into the dorsal part of the spinal cord, the impaled fiber showed a progressive deterioration in its ability to develop action potentials. Records $A$ to $D$ in fig. 13 illustrate this. A notch

FIG. 13. Progressive deterioration in the action potentials from a fiber in the dorsal funiculus ( $A$ to $D$ ) and restoration of the response by constant currents applied through the recording micro-electrode. Time marker, 1000 cycles per sec.
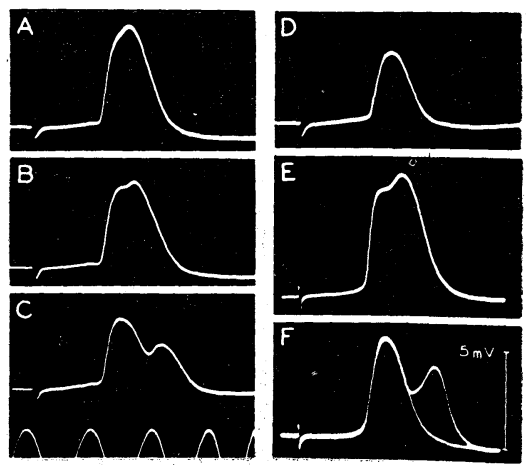

in the course of action potential, which was just visible at the beginning, became clearer and clearer as time elapsed. About three minutes after the start of the observation, the elevation following the notch began to drop out once in every two to three sweeps. According to the results of the experiments made on excised motor nerve fibers, this change in the shape of the action potential indicated block of conduction across the impaled region.

When the conduction was completely blocked, a constant current of 3 to $20 \times 10^{-9}$ ampere was sent into the fiber through the micro-electrode (see Method section) in such a direction that the surface membrane of the fiber could be re-polarized by the current. Just as in fibers in the peripheral nerve, this procedure brought the nerve fiber to the initial or a still better state. As time elapsed, the intensity of current needed for restoration of conduction increased gradually. This property again was common to the fibers in the peripheral nerve and those in the spinal cord.

When the micro-electrode inserted into the spinal cord was giving action potentials without associated resting potential, the passage of a current of the intensity mentioned above increased the noise level of the oscillograph record but generally no appreciable increase in the spike height was observed. From our experience with the isolated single nerve fiber, we believe that the tip of the micro-electrode was covered, on such occasions, with a thin film of myelin.

It is interesting to note that, in anodal polarization of a single fiber in the dorsal funiculus with an internal electrode, more than one notch can appear in 


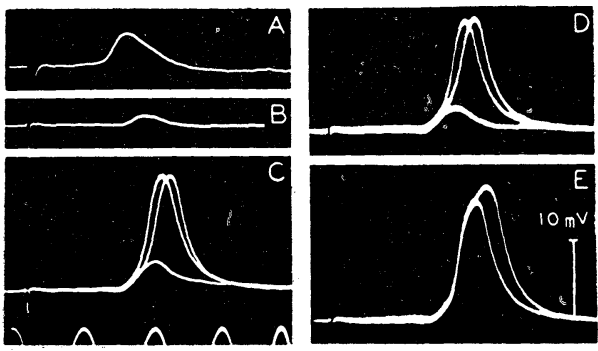

FIG. 14. Same as fig. 13. Note the number of the steps in the largest response in record $E$. Time marker, 1000 cycles per sec.

action potential records. An example of such records is furnished in fig. 14. Records $A$ and $B$ in this figure show the progressive decline in the spike height which followed the appearance of a resting potential (about $20 \mathrm{mV}$ in this case). Then the fiber was re-polarized with a constant current of 5 to $10 \times 10^{-9}$ amperes, and at various intensities two to four successive sweeps of the oscillograph were photographed on one frame of film. From these superimposed photographs, it seems to me safe to conclude that the highest action potential in record $D$ shows three discontinuities (in its slope), or three notches.

In 1934, Erlanger and Blair made a similar observation on fibers in the peripheral nerve and stressed the importance of the nodes of Ranvier in nervous conduction under anelectrotonus. Now we have strong evidence for believing that the process of nervous conduction in the fibers in the spinal cord is similar to that in the peripheral nerve.

3. Measurement of threshold along individual fibers in the dorsal funiculus. Ono (1934), working under direction of Kubo, demonstrated that the threshold for an excised single nerve fiber, measured with an electrode of 10 to 100 microns in diameter that was moved along the fiber, shows a sharp minimum at every node of Ranvier. Since all the experiments described so far attest to a striking similarity between fibers in the sciatic nerve and those in the dorsal funiculus, it seemed interesting to find whether or not individual fibers near the surface of the dorsal column of the spinal cord show a regular variation in threshold like the fibers in peripheral nerves.

The movable electrode used for this purpose was a glass capillary having a tip of approximately 25 microns inside diameter and 40 microns outside diameter. The tip of this capillary was smoothed by grinding it on a fine oilstone. This precaution was taken to avoid inflicting mechanical injury on the

FIG. 15. Single-fiber responses recorded from a few fibers entering the gastrocnemius muscle were evoked by stimulating the dorsal surface of the frog spinal cord. The two lower records were obtained with the stimulating electrode placed on a "sensitive spot" near the entry of the 8th dorsal root; the two upper records from another sensitive spot situated at a distance of $4.2 \mathrm{~mm}$. proximal to the first sensitive spot. Time marker, msec.

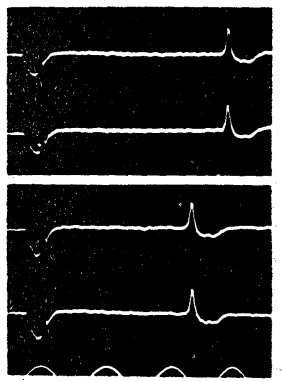


nerve fibers in the cord when the electrode was moved along the surface of the pia. Stimulating currents, rectangular pulses of approximately $0.1 \mathrm{msec}$. duration in most cases, were always applied through the intact pia. The stimulating electrode was connected to the cathode of a square wave generator. The anode of the stimulating circuit was in the bath of Ringer's fluid in which the spinal cord was immersed. The index of excitation was single fiber responses (fig. 15) from the nerve fibers entering the gastrocnemius muscle by way of the dorsal roots (see Method).

It was possible to demonstrate by this method that the threshold for an individual fiber in the dorsal funiculus shows a marked variation along its course. In favorable cases the course of the fiber could be traced for a stretch of $3 \mathrm{~mm}$. or even more (see fig. 16); the points at which the stimuli applied through the glass capillary gave singlefiber responses were found to lie on a long straight line, and all these highly sensitive spots gave responses in one and the same single fiber (see fig. 15). The spacing between these sensitive spots was in general fairly regular. It was usually between 200 and 300 microns; but distances of about 400 microns or more were also encountered in some preparations (in the frog). The point of interest in these experiments is that at the point half-way between these highly sensitive spots the fiber was practically insensitive to the stimulating current.

The absolute value of the current needed for this stimulation is also of some interest. For pulses of $0.1 \mathrm{msec}$. duration, the threshold intensity ranged from 1.5 to 4.5 microamperes. This value is slightly less than the current intensity required for excitation of a single excised nerve fiber immersed in a volume of Ringer's fluid (Tasaki, 1950).

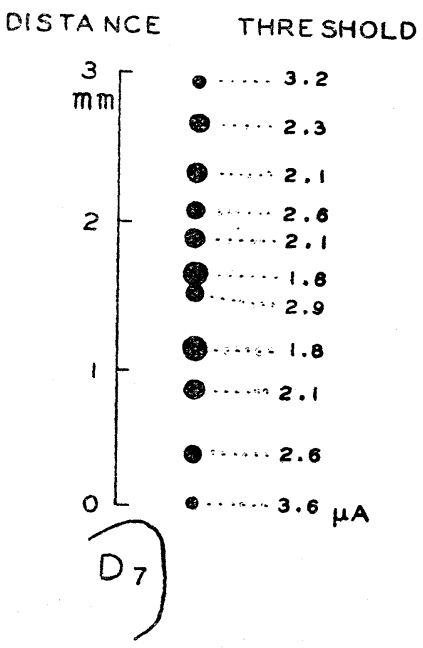

FIG. 16. Distribution of the "sensitive spots" on the dorsal surface of a bull frog spinal cord. Single-fiber responses in the muscle nerve were taken as the index of excitation.

The classical theory of electricity tells us that the potential $V$ at the tip of a glass capillary of radius $r$ through which a current of the intensity $I$ is flowing is given approximately by

$$
V=\frac{I S}{4 \pi r},
$$

where $S$ is the specific resistance of the fluid medium. According to the argument developed previously, the potential $V$ at the tip of a capillary, which is in contact with a node, should exceed, in order to stimulate the fiber, approximately one half of the threshold value obtained by the method of bridge-insulators. The rheobase of a single fiber mounted on bridge-insulator is generally between 25 and $40 \mathrm{mV}$ regardless of the fiber type; it is slightly greater in small fibers than in large ones and for shorter pulse duration it invariably 
tends to become larger. According to this argument based on the data taken from isolated single nerve fibers, therefore, the threshold voltage $V$ at the tip of the electrode should be of the order of $30 \mathrm{mV}$. Introducing this value and the specific resistance of frog ringer for $S$, namely $10^{2} \mathrm{ohm}-\mathrm{cm}$., we find that the threshold intensity of the current $I$ measured with a glass capillary of radius $r$ placed directly on a node should be approximately

$$
I \fallingdotseq 4 r \text { milliamperes. }
$$

For a capillary having a tip of 25 microns diameter, namely $r=1.25 \times 10^{-3} \mathrm{~cm}$, $I$ should be approximately 5 microamperes. This calculated value agrees roughly with the threshold obtained by the direct measurements mentioned above.

In the simplified argument above, we assume the fiber in the dorsal funiculus to be imbedded in a continuous conducting medium with uniform specific resistance. Undoubtedly, the white matter of the spinal cord has a specific resistance greater than that of frog ringer. This situation probably accounts for the fact that the flbers in the dorsal funiculus show a threshold value a little lower than the calculated value. Thus it seems to me safe to conclude that the sensitive spots along the fiber in the dorsal funiculus show practically the same threshold value as the nodes of Ranvier of the fibers in the sciatic nerve.

The situation that the specific resistance (or rather the impedance) of the spinal cord is larger than that of the Ringer's fluid in which the cord is immersed tends to make the potential field generated by the stimulating current in the cord decay more sharply than in a uniform conducting medium and consequently to make the variation of threshold along the fiber more pronounced. Actually, the threshold at the point half way between two neighboring sensitive spots was so high that it was practically impossible to get a response at all. The current intensity was varied by steps of $1 \mathrm{db}$. (11 per cent), and, to avoid unduly strong stimulation, it was not allowed to exceed $12 \mathrm{db}$. (400 per cent) above the threshold for the sensitive spots. In a certain stretch between two sensitive spots, this intensity was found to be ineffective. In the corresponding experiments on the peripheral nerve fiber, the threshold along the fiber varied more than $30 \mathrm{db}$. (Tasaki, 1950). A similar result was obtained by Lussier and Rushton (1952) on individual flbers in an intact peripheral nerve.

By the technique described above it was not possible to trace the course of a single fiber in the dorsal funiculus down to a spinal root. The threshold was higher near the two ends of a series of sensitive spots, and no response was obtained from outside this region. This indicates that the fiber under investigation no longer stayed in the most superficial layer at both ends of the sensitive region. Sometimes a tendency was seen that the course of the fiber approached gradually to the mid-line of the spinal cord as it went upwards in the dorsal funiculus.

Few attempts were made to estimate the conduction rate of the fibers in the dorsal funiculus. Since the maximum length available in the cord was only 2 to $4 \mathrm{~mm}$., the measurement was not accurate; but, for fibers having a rate of about $25 \mathrm{~m} / \mathrm{sec}$. in the sciatic nerve, the average rate in the cord was found to be slightly less than $10 \mathrm{~m} / \mathrm{sec}$. 
Most of these threshold measurements were made on small green frogs, but a few experiments done on larger bull frogs indicated that for this kind of experiment the larger frogs are better than the smaller ones.

\section{DISCUSSION}

The experimental results described in Part I of this paper hardly require any comment. All these new data confirm and substantiate the conclusions we had reached previously (Tasaki and Takeuchi, 1941 and 1942). The nodes of Ranvier are only places in the myelinated nerve fiber at which the process of excitation can occur. The myelin sheath or a layer of myelin shows a high resistance to direct currents, but transient current pulses can flow through this layer. A pulse of outward-directed current sent through the nodal membrane, or through a part of this membrane, can elicit a large response which raises the potential of the axoplasm inside this membrane for a period of about $1 \mathrm{msec}$. This positive variation in the potential of the axoplasm spreads along the cable-like structure of the internode. In a normal nerve fiber this wave causes a strong outward directed current through the adjacent node of Ranvier, strong enough to excite it in a very short time. This process of restimulation by the action current is generally referred to as the saltatory conduction (see also Tasaki and Mizuguchi, 1948; Huxley and Stämpfli, 1949).

The term "saltatory" caused a misunderstanding among some physiologists (e.g. Bullock, 1951 ; Woodbury, 1952 ; Laporte, 1952). It is not correct to interpret this term as meaning that an impulse jumps across each internode at an almost infinite speed. On the contrary, a large part of the internodal conduction time is spent in the above-mentioned spread of the potential wave along the internode (Hodler, Stämpfli and Tasaki, 1952). One of the conclusions in Huxley and Stämpfli's paper (1949), "the conduction time of the longitudinal action current is constant in each internode," is not quite correct.

The experimental results mentioned in Part II give us very strong physiological evidence against the belief that there are no nodes of Ranvier in the myelinated fibers in the central nervous system ( $c f$. Grundfest, 1937). Histological evidence against this wide-spread belief was presented recently by Huxley and Stämpfli and by Hess and Young (1949). According to Hess and Young, the internodal length of the fibers in the spinal cord is proportional to the fiber diameter, just as in peripheral nerves. The relatively short internodal dis tance in the fibers of the dorsal funiculus and the low conduction rate agree, at least qualitatively, with the histological finding that the fibers in this part of the spinal cord are smaller than in their path in the peripheral nerves.

\section{CONCLUSIONS}

1. Using micro-electrodes, resting and action potentials were recorded from inside isolated myelinated nerve fibers. The observed resting potential varied from zero to $30 \mathrm{mV}$, and the peak value of the action potential was less than $30-40 \mathrm{mV}$. These low values are attributed to mechanical injury of the fibers by the electrode. 
2. Action potentials of 1 to $30 \mathrm{mV}$ (peak value) were frequently recorded when no resting potential was observed with the micro-electrode inserted into the axis-cylinder. This phenomenon is ascribed to the high DC resistance of a thin film of myelin that is assumed to have covered the tip of the electrode.

3. Difficulties in recording action potentials from inside small myelinated fibers were encountered and are discussed.

4. By polarizing single nerve fibers in the dorsal funiculus, physiological evidence was secured that show the presence of nodes of Ranvier in the central nervous system.

5. The threshold for a single fiber in the dorsal funiculus was found to vary considerably along its course. The nodes of Ranvier of the fiber could be located by this method.

6. The myelinated nerve in the central nervous system are very similar to those in the peripheral nerves.

My thanks are due to Dr. Hallowell Davis for his help in preparing this paper for publication.

\section{REFERENCES}

1. Bullock, T. H. Ann. Rev. Physiol. 13 : 261-280, 1951.

2. Castillo-Nicolau, J. Del AND StTaris, L. J. Physiol. 114 : 19 P, 1951.

3. ERlanger, J. AND Blair, E. A. Am. J. Physiol. 110: 287-311, 1934.

4. Grundfest, H. Ann. Rev. Physiol. 9: 477-506, 1947.

5. Hess, A. AND Young, J. Z. Nature 164: 490-493, 1949.

6. Hodgkin, A. L. Proc. Roy. Soc. B. $126: 87-121,1938$.

7. Hodgkin, A. L., Huxley, A. F. AND Katz, B. Arch. Sc. Physiol. 3 : 129, 1949.

8. Hodler, J., Stämpfli, R. ANd TASAki, I. Am. J. Physiol. in press.

9. Huxley, A. F. AND Stämpfli, R. J. Physiol. 108: 315-339, 1949; $112: 476-495,1951$.

10. HUXLEY, A. F. AND TASAKI, I. in preparation.

11. KATO, G. Cold Spring Harbor Symposia on Quantitative Biology 4: 202-213, 1936.

12. LAporte, Y. J. Gen. Physiol. 35 : 323-342, 1951.

13. Ling, G. ANd Gerard, R. W. J. Cell. Comp. Physiol. 34: 382-396, 1949.

14. Lussier, J. J. AND Rushton, W. A. H. J. Physiol. $117:$ 87-108, 1952.

15. Nastuk, W. L. ANd Hodgkin, A. L. J. Cell. Comp. Physiol. 35: 39-73, 1950.

16. ONO, S. IN KATO, G. Microphysiology of Nerve, Tokyo, 1934.

17. Rushton, W. A. H. Proc. Roy. Soc. B. 124: 210-243, 1937.

18. Schoeffle, G. M. AND ERlanger, J. Am. J. Physiol. $167:$ 134-146, 1951.

19. TASAKI, I. Am. J. Physiol. $125: 367-379,1939$.

20. TASAKI, I. AND KANO, H. Japan. J. Med. Sci. III Biophysics 9: 17, 1942.

21. TASAKI, I. AND Mizuguchi, K. J. Neurophysiol. $11:$ 295-303, 1938.

22. TASAKI, I. AND TAKEUChI, T. Pfügers Arch. f. ges. Physiol. 244: 696-711, $1941 ; 245$ :

764-771, 1942.

23. Woodbury, J. W. J. Cell. Comp. Physiol. 39 : 323-339, 1952. 